\title{
Why is the new journal Soft Science needed?
}

\author{
Chuanfei Guo ${ }^{1}$, Cunjiang Yu², Zhifeng Ren ${ }^{3}$ \\ 'Department of Materials Science and Engineering, Southern University of Science and Technology, Shenzhen 518055, \\ Guangdong, China. \\ ${ }^{2}$ Department of Mechanical Engineering, University of Houston, Houston, TX 77204, USA. \\ ${ }^{3}$ Department of Physics and Texas Center for Superconductivity at the University of Houston (TcSUH), University of Houston, \\ Houston, TX 77204, USA.
}

Correspondence to: Prof. Zhifeng Ren, Department of Physics and Texas Center for Superconductivity at the University of Houston (TcSUH), University of Houston, Houston, TX 77204, USA. E-mail: zhifengren2013@gmail.com

How to cite this article: Guo C, Yu C, Ren Z. Why is the new journal Soft Science needed? Soft Sci 2021;1:7. https://dx.doi.org/10.20517/ss.2021.12

Received: 16 Aug 2021 Accepted: 16 Aug 2021 Available online: 18 Aug 2021

Academic Editor: Zhifeng Ren Copy Editor: Xi-Jun Chen Production Editor: Xi-Jun Chen

Research on mechanically deformable, flexible, or stretchable (i.e., "soft" as a general term) materials and devices is a fast-growing field with numerous exciting new developments in fundamental science and emerging applications enabled by new materials, advanced fabrication processes, and novel device technologies. This soft materials and devices research has drastically impacted healthcare, medicine, robotics, space exploration, and the internet of things ${ }^{[1-3]}$. For example, soft e-skins can enable robots to achieve tactile sensation, and flexible wearables and implantables can help monitor the health of the human body $^{[4-6]}$.

The rising interest in soft materials and electronic devices research is also evidenced by the thousands of papers that have been published in this field, with an annual growth rate of $\sim 20 \%$ over the past five years. These publications have also generated an unusually high impact. For example, a survey of recent papers on flexible electronics showed that these papers had contributed significantly to the impact factors of the journals in which they were published [Figure 1], although they make up no more than $10 \%$ of the total publication in any single journal [Figure 2].

It is envisioned that research on soft materials, devices, and systems will continue to thrive in the coming decades in both academia and industry. The already large, fast-growing community has called for

The Author(s) 2021. Open Access This article is licensed under a Creative Commons Attribution 4.0 International License (https://creativecommons.org/licenses/by/4.0/), which permits unrestricted use, sharing, adaptation, distribution and reproduction in any medium or format, for any purpose, even commercially, as long as you give appropriate credit to the original author(s) and the source, provide a link to the Creative Commons license, and indicate if changes were made. 


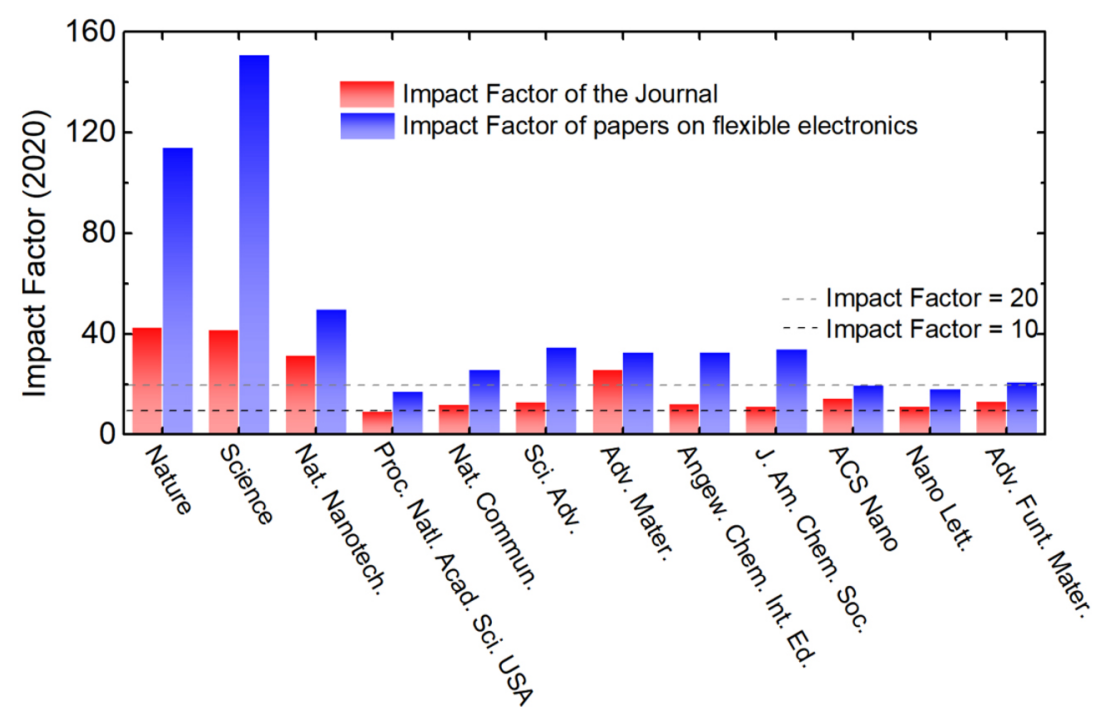

Figure 1. Impact factors of representative journals and papers on flexible electronics published in these journals.

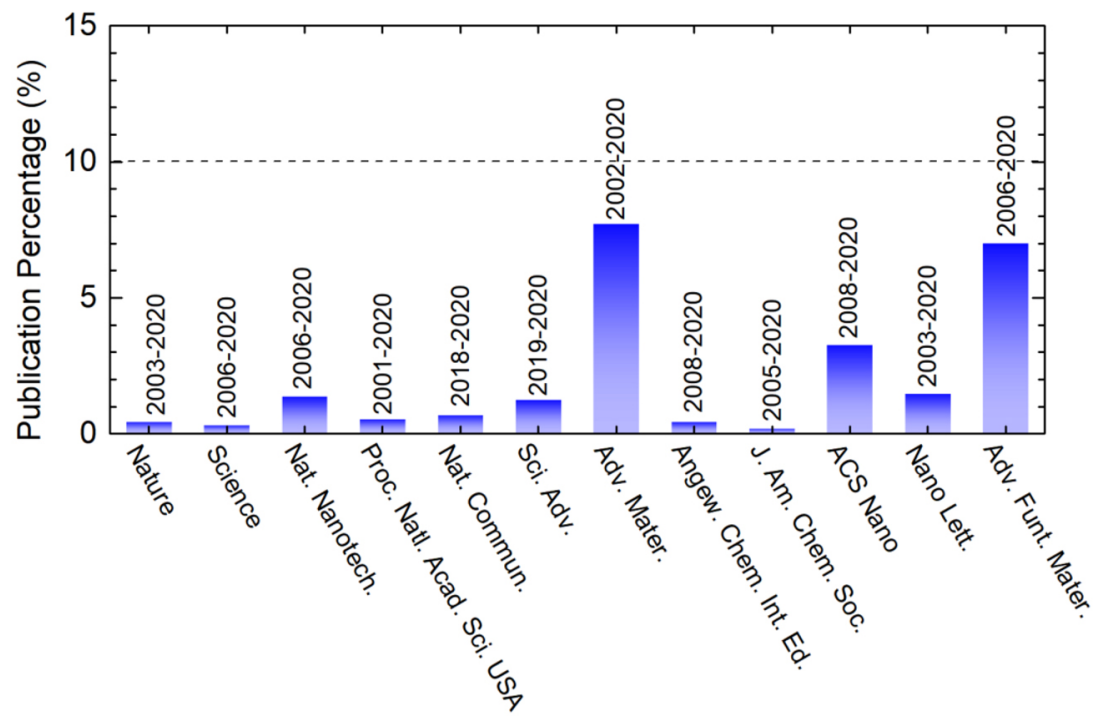

Figure 2. Publication of papers on flexible electronics as percentages of all published papers in representative journals.

specialized journals focusing on soft devices and systems research. In 2016, Nature Publishing Group launched npj Flexible Electronics, the first journal specializing in "flexible electronic systems, including plastic electronics and emerging materials, new device design and fabrication technologies" ${ }^{\text {"[] }}$. It received its first impact factor of 12.7 in 2021 and is expected to be a flagship journal for this emerging field. However, there exists no other journal specializing in soft devices and systems.

To better accommodate the needs of this fairly large and fast-growing community, we feel that it is very timely to launch such a specialized journal. We have therefore launched this new journal, Soft Science, which is aimed to become a high-profile and specialized platform that publishes high-quality papers on mechanically soft systems on a variety of scales, ranging from materials (e.g., hydrogels) to devices (e.g., flexible electronics) to functional systems (e.g., soft robotics). This journal has received its first batch of submissions, and six high-quality papers have been published in its first issue. These three reviews and three 
research articles cover flexible tactile sensors, wearable supercapacitors, elastomers, and organic/inorganic thermoelectric materials.

As the editorial team of a new journal, we promise to handle all the submitted papers very quickly so that a decision can be reached in a time much shorter than most of the other journals and published online within $24 \mathrm{~h}$ after acceptance.

\section{DECLARATIONS}

Author's contributions

Preparation of the manuscript: Guo C, Yu C, Ren Z

\section{Availability of data and materials}

Not applicable.

\section{Financial support and sponsorship}

None.

\section{Conflicts of interest}

All authors declared that there are no conflicts of interest.

\section{Ethical approval and consent to participate}

Not applicable.

\section{Consent for publication}

Not applicable.

\section{Copyright}

(c) The Author(s) 2021.

\section{REFERENCES}

1. Wang X, Liu Z, Zhang T. Flexible Sensing Electronics for wearable/attachable health monitoring. Small 2017;13:1602790. DOI PubMed

2. Rich SI, Wood RJ, Majidi C. Untethered soft robotics. Nat Electron 2018;1:102-12. DOI

3. Kang J, Tok JB, Bao Z. Self-healing soft electronics. Nat Electron 2019;2:144-50. DOI PubMed

4. Wang S, Xu J, Wang W, et al. Skin electronics from scalable fabrication of an intrinsically stretchable transistor array. Nature 2018;555:83-8. DOI PubMed

5. Someya T, Bao Z, Malliaras GG. The rise of plastic bioelectronics. Nature 2016;540:379-85. DOI PubMed

6. Gao W, Ota H, Kiriya D, Takei K, Javey A. Flexible Electronics toward Wearable Sensing. Acc Chem Res 2019;52:523-33. DOI PubMed

7. npj Flexible Electronics. Available from: https://www.nature.com/npjflexelectron/about. [Last accessed on 16 Aug 2021]. 